\section{P89 TOXIC EPIDERMAL NECROLYSIS IN A 4 YEAR OLD GIRL IN THE UAE}

Alyazia Al Hallami* ${ }^{*}$ Najla Al Kuwaiti, Omer Yousef. Tawam hospital, Abu Dhabi/Alain, UAE

10.1136/archdischild-2019-epa.444

Learning objectives Present a case of toxic epidermal necrolysis in the UAE who was found to have mycoplasma pneumoniae respiratory infection.

Case summary A 4-year-old girl, previously healthy, presented with fever of 3 days with a rash for 1 day. The rash was erythematous, sand paper like involving her chest, back, upper and lower extremities. She was found to have streptococcal pharyngitis, treated as a case of scarlet fever and discharged home on Amoxicillin-Clavulanate acid and ibuprofen.

The next day, she presented again with fever and increasing rash that is now involving the eyes and mouth (lips an oral mucosa). The rash was evolving to erosions and sloughing of the skin on the chest and back.

Considering that her skin involvement was tremendous (> $30 \%$ ), she was diagnosed as toxic epidermal necrolysis (TEN). She was admitted to the pediatrics intensive care unit (PICU).

When looking for the underlying cause, the triggering factor was suspected to be either drug related or infection related. She was tested for mycoplasma infection that turned out to be positive, and she was managed with azithromycin.

Throughout her illness she was hemodynamically stable, on the $4^{\text {th }}$ day of illness she started to improve in sense of settling fever and tachycardia, Her inflammatory markers were decreasing as well.

She continued her medical care in the hospital for 20 days and discharged home with follow-ups.

Methods Case report and literature review

Discussion We believe as clinicians that in the UAE we rarely encounter such critical cases that warrant a multidisciplinary approach, aiming to deliver the best care and maintain the well being of the patient. In reviewing the literature there was no reported case in the UAE. Moreover, etiological underlying factors are clearly known in the medical history. We believe that three factors were implied in this case; Amoxicillin use, mycoplasma pneumoniae respiratory infection and the possibility of genetic predisposition.

Conclusion SJS/TEN is a challenging diagnosis, that merits prompt recognition and management hoping to prevent its sequelae. Many factors contribute to the development of these unpleasant and eventful diagnoses. Pediatricians need to be alerted regarding this diagnosis and the available management modalities.

\section{P90 CONSTRICTIVE PERICARDITIS: A RARE AND CHALLENGING DIAGNOSIS}

${ }^{1}$ Vasanthee Sundram*, ${ }^{1}$ Aimee Cooper, ${ }^{2}$ Des Cox, ${ }^{2}$ Patrick Gavin, ${ }^{2}$ Adam James, ${ }^{1}$ Edwina Daly. ${ }^{1}$ Tallaght University Hospital, Dublin, Ireland; ${ }^{2}$ Our Lady's Children's Hospital Crumlin, Dublin, Ireland

\subsection{6/archdischild-2019-epa.445}

Constrictive pericarditis is a rare condition in children and often presents with clinical features unrelated to the heart making diagnosis challenging. It results from scarring leading to inelasticity of the pericardial sac which produces an inability to adapt to volume changes due to a restriction in diastolic filling. The most common causes in the developed world are idiopathic, prior cardiac surgery and chest radiotherapy. In the developing world tuberculosis remains the commonest cause.

We herein report an eleven year old boy of Indian ethnicity who attended a Dublin Paediatric Emergency with chest pain and shortness of breath.

$\mathrm{He}$ was living in Ireland but spent the first five years of his life in India. His vaccinations including BCG were up-todate. There was no history of contact with Tuberculosis.

$\mathrm{He}$ was afebrile and had a normal respiratory rate, heart rate and heart sounds. Examination revealed dullness on percussion in the left lower lobe of the lung with tender hepatomegaly. Chest radiograph reported dense consolidation in the left lower lobe with a parapneumonic effusion.

$\mathrm{He}$ had a low serum albumin and total protein at $22 \mathrm{~g} / \mathrm{L}$ and $46 \mathrm{~g} / \mathrm{L}$ respectively and elevated gamma-glutamyl transferase of $80 \mathrm{U} / \mathrm{L}$.

The patient was treated with intravenous Cefotaxime and oral Azithromycin for possible pneumonia. He remained systemically well but a repeat radiograph taken five days later showed no improvement.

Analysis of pleural fluid by thoracentesis indicated a transudative type. He was seen by the Infectious Disease team and all investigations including inflammatory markers were normal. An eye exam was also normal.

Chest computed tomography (CT) scan reported mild pericardial thickening with hepatic enlargement. A large left-sided pleural effusion with minor basal atelectasis and a small contralateral pleural effusion was noted. There was small mediastinal lymphadenopathy.

Electrocardiogram (ECG) showed flattening of the T-waves and was generally low voltage.

Echocardiogram revealed dilated inferior vena cava and hepatic veins with thickened pericardium and intra-atrial septum deviating towards the Left Atrium suggesting elevated Right Atrial pressure.

Cardiac catheterisation demonstrated elevated pressures consistent with constrictive pericarditis. The patient has been taken over by the cardiology team for ongoing management.

Discussion Constrictive Pericarditis is a rare condition in children but this case highlights the importance of a meticulous and multidisciplinary approach to look at other causes when a patient is not responding to treatment as expected.

\section{P91 'STRIDOR' THE PRESENTING SYMPTOM OF SCIMITAR SYNDROME WITH TRACHEAL RING - A CASE REPORT}

Wajida Mazher*, John Twomey. University Hospital Limerick, Limerick, Ireland

\subsection{6/archdischild-2019-epa.446}

We report a very interesting case of Scimitar syndrome with tracheal ring in a 5 month old thriving baby with chronic stridor.

Stridor is caused by upper airway obstruction and laryngomalacia being the most common cause of chronic stridor in children, followed by croup, which is an acute presentation.

Scimitar syndrome on the other hand is a rare congenital cardiopulmonary anomaly with incidence of 2 per 100,000 live births with 2:1 female predominance, consists of partial anomalous pulmonary venous connection of right lung to inferior vena cava, right lung hypoplasia , dextrocardia and anomalous systemic arterial supply to right lung. 
Our patient presented with worsening stridor over time and required intubation due to respiratory failure. Echocardiogram, CT angiogram and MRI revealed Scimitar syndrome with PAPVD (Partial anomalous pulmonary venous drainage) and tracheal ring. MAPCAs (Major aortopulmonary collateral arteries) ligation, PAPVD repair, laryngeal repair and slide tracheoplasty was successfully done and baby showed significant clinical improvement.

\section{P92 EPIDIDYMITIS IN HENOCH-SCHONLEIN PURPURA, AN UNUSUAL PRESENTATION OF A COMMON VASCULITIC CONDITION IN CHILDREN}

Muhammad Imran Riazat ${ }^{\star}$, Birendra Rai, Farhana Sharif. Mullingar Regional Hospital, Mullingar, Ireland

\subsection{6/archdischild-2019-epa.447}

Background Henoch-Schonlein purpura is a common vasculitic condition in paediatric age group. The hallmark of this syndrome is vasculitic skin rash, arthralgia and abdominal pain. Acute scrotal pain and swelling is not a common presentation of this disease. We would like to present a six and half yearold boy who presented with pain and left testicular swelling. We suspected epididymitis and torsion of testes clinically. Torsion was ruled out by doing doppler ultrasound. He was successfully managed conservatively by oral steroids and analgesia. Epididymitis is more common than torsion in HSP. Aim of this case presentation is to improve awareness of this unusual morbidity of this common vasculitic condition in children.

Case report A six and half year-old Irish, Caucasian boy presented to our emergency room with one day history of abdominal pain, rash on legs and followed by scrotal pain and swelling. There was no preceding history of any illness prior to his presentation. According to his parents he developed rash on his lower legs a day prior to hospital visit. He started complaining of pain in this mid abdomen followed by pain in his left scrotum. There was no history of vomiting, diarrhoea or bleeding from any site of his body. They denied history of sick contact before this illness. There were no previous medical complaints.

His examinations revealed normal vital signs. He had nonblanching purpuric rash on his lower limbs and feet. His abdominal examination showed soft non- tender abdomen with no guarding. He had scrotal swelling and was complaining of left scrotal pain. Routine blood investigations performed including FBC, coagulation profile, $\mathrm{U} \& \mathrm{E}$, blood culture. The results turned out to be normal. A diagnosis of Henoch-Schonlein purpura was made. Urgent surgical review was requested. He was seen by surgical team and was diagnosed epididymitis clinically. An US doppler of scrotum requested as part of clinical workup reported normal results.

As his condition failed to improve, we started him a short course of oral steroids along with analgesia. He showed significant improvement and was discharged after 2 days to complete treatment at home.

Conclusion Henoch-Schonlein purpura can cause immune mediated epididymitis and painful scrotum. Patient can be managed conservatively if US scrotal region is normal and surgical exploration can be avoided.

\section{P93 THE MEDICAL AND SURGICAL MANAGEMENT OF RAISED INTRAOCULAR PRESSURE (IOP) IN CHILDREN: A CHALLENGING OPHTHALMOLOGY CASE}

${ }^{1}$ Deirdre Ryan*, ${ }^{2}$ Stephen Farrell. ${ }^{1}$ Temple St Children's University Hosptial, Dublin, Ireland; ${ }^{2}$ Ophthalmology Department, Temple St Children's University Hosptial, Dublin, Ireland

\subsection{6/archdischild-2019-epa.448}

Background Glaucoma is one of the most common causes of blindness in adults. In children, while it is much less common it is even more challenging to diagnose, monitor and treat.

Case 7 year old SA presented with headache on a background of Eczema and Vernal Keratoconjunctivitis. Full work up was normal, including Lumbar Puncture and CT Brain. He was poorly compliant with topical steroids prescribed for a Keratoconjunctivitis flare. On examination during Ophthalmology review, SA had conjunctival injection with corneal punctate erosions consistent with ocular surface disease. Exam was otherwise normal but difficult to perform due to photosensitivity. Symptoms improved with topical steroids, which were tapered with close monitoring as an outpatient. At two months IOPs were elevated. Steroids were discontinued and pressure reducing drops commenced. Mild hypertension was measured on subsequent visits.

SA re-presented with acute photophobia and left frontal headache. IOPs were elevated at $60 \mathrm{mmHg}$ (left) and 45 $\mathrm{mmHg}$ (right). Acetazolamide and Mannitol were administered intravenously. During examination under anaesthetic (EUA), pressure in left eye measured $34 \mathrm{mmHg}$ and normal in right. The sclerae were extremely hardened. Pigment and fibrin were noted in the left anterior chamber. Left cup:disc ratio was 0.3 , significantly greater than 0.1 in the right, with normal fundi. He was diagnosed with scleritis and anterior uveitis. Investigations for causes of uveitis were all negative; including Anterior Chamber Paracentesis (Herpes Simplex), MRI Brain (Vasculitis), Quantiferon Gold (Tuberculosis), Syphilis, Lyme and Bartonella serology, ANCA, serum ACE, Chest XRay. Treatment comprised oral Prednisolone, topical steroids, Acetazolamide, Latanoprost, Timolol and Dorzolamide. Inflammation resolved, pressure was reasonably controlled. Glaucoma surgery is likely to fail in the presence of active or recent inflammation, so surgical management was postponed.

Despite maximum medical management SA presented a third time with raised IOP. $\mathrm{He}$ was brought to theatre urgently. Ahmed Valve was inserted into his left eye. Bilateral scleral calcification led to difficult valve insertion. His formal diagnosis is Idiopathic Nodular Scleritis, Intraocular Inflammation and Left Uveitic Glaucoma.

Conclusion Management of childhood glaucoma and uveitis is challenging especially in rare conditions such as this. SA's IOP is controlled, although accurate monitoring remains challenging. Rigid thickened corneas lead to falsely elevated pressures. SA developed astigmatism due to nodular scleritis involving the peripheral cornea. No progression of optic disc cupping was noted at further EUA. Despite a difficult clinical course, SA is currently asymptomatic on tapering doses of steroids and Adalizumab, enjoying an improved quality of life. 